INRA Prod. Anim., 2005, 18 (3), 169-173

\section{Production et qualité nutritionnelle des phosphates alimentaires}

W. BLEUKX

Tessenderlo Group Technical Manager Feed Ingredients, Rue du Trône 130, B-1050 Bruxelles

Courriel : wouter.bleukx@tessenderlo.com
La majorité des régimes alimentaires distribués aux animaux de rente ne permet pas une couverture satisfaisante de leurs besoins en phosphore $(\mathrm{P})$, surtout dans des conditions de production intensives ; par ailleurs, il devient nécessaire de mieux contrôler les rejets en $\mathrm{P}$ dans les effluents d'élevage. Ces questions trouveront leurs solutions dans une meilleure adéquation des recommandations d'apport alimentaire de $P$ pour les diverses espèces d'animaux d'élevage et par l'utilisation de sources d'apport complémentaire (principalement les phosphates inorganiques) de bonne qualité nutritionnelle. Cet article se propose, après avoir rappelé les principales technologies de la production des phosphates alimentaires pouvant conduire à des sources de $\mathrm{P}$ sensiblement différentes, d'analyser les critères de qualité des phosphates utilisés en alimentation animale.

\section{1 / La production des phosphates alimentaires}

Plusieurs types de phosphates alimentaires de différentes origines sont produits dans l'Union Européenne, les principaux sont le phosphate bicalcique anhydre $\left(\mathrm{DCPOH} \mathrm{H}_{2} \mathrm{O}\right)$, obtenu par neutralisation de l'acide phosphorique défluoré par une source de calcium (figure 1), et le phosphate bicalcique cristallin dihydraté $\left(\mathrm{DCP} 2 \mathrm{H}_{2} \mathrm{O}\right)$. La production de DCP2 $\mathrm{H}_{2} \mathrm{O}$ commence par la dissolution du phosphate brut dans l'acide chlorhydrique entraînant la formation d'une solution de phosphate monocalcique après purification, la précipita-
Figure 1. La production de phosphate bicalcique avec l'acide phosphorique.

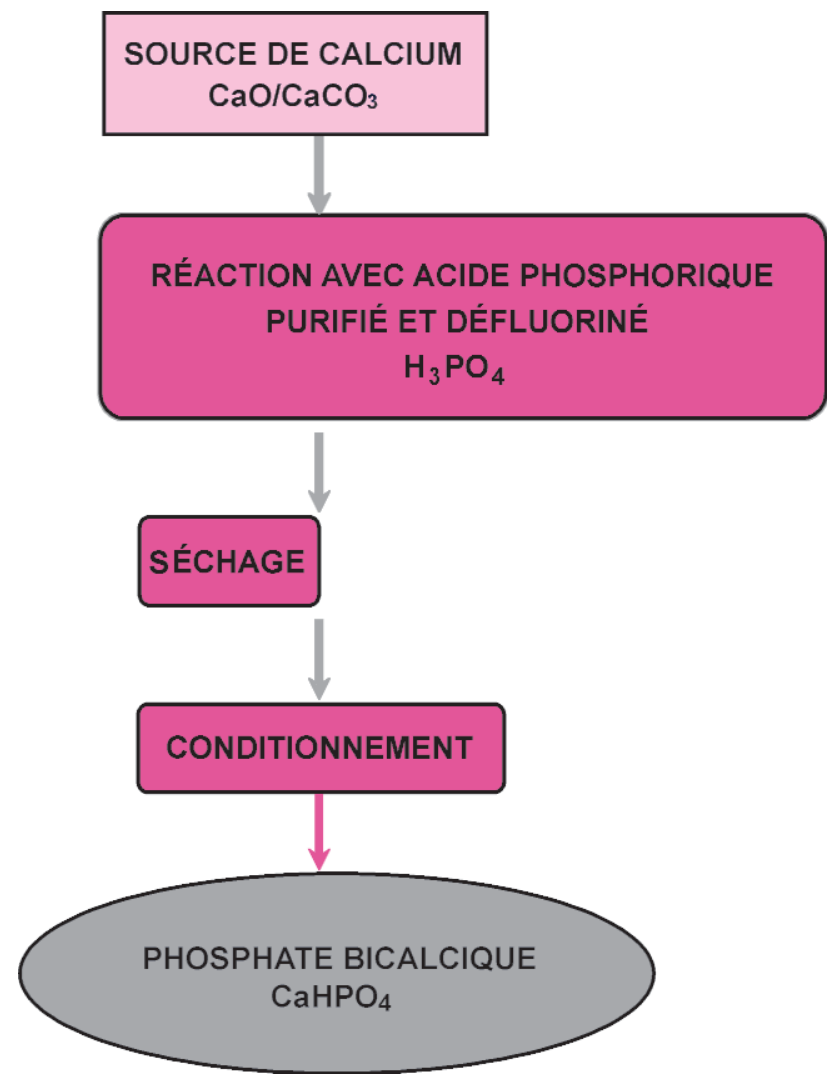

tion de cette solution par une source de calcium permet d'obtenir les cristaux de phosphate bicalcique dihydraté (figure 2). L'ensemble des phosphates bicalciques représente approximativement $60 \%$ de la consommation de phosphates alimentaires dans l'UE. D'autres sources sont également utilisées principalement le phosphate monocalcique (MCP), le phosphate monobicalcique (MDCP) et à un moindre degré les phosphates de magnésium, calcomagnésien et mono ou di-ammoniques. Tous ces phosphates sont produits à partir d'une source de calcium, et/ou de magnésium ou d'ammonium et de l'acide phosphorique alimentaire défluoré et purifié. Le MCP peut également être produit à partir du phosphate bicalcique (figure 3), le $\mathrm{DCP} 2 \mathrm{H}_{2} \mathrm{O}$ permet d'obtenir un MCP pur et stable. Le phosphate naturel défluoré (DFP) est également disponible mais n'est pas produit dans l'UE. Il est obtenu par chauffage, à des températures supérieures à $1200^{\circ} \mathrm{C}$ d'un mélange de phosphate brut, d'acide phosphorique et d'une source de sodium. Le DFP est parfois défini, à tort, comme un phosphate de calcium et de sodium (CaNaP) mais il ne contient que peu de $\mathrm{CaNaP}$, la plus grande partie (environ $70 \%$ ) étant sous forme tricalcique.

\section{2 / Consommation des phosphates alimentaires}

La consommation de phosphates alimentaires dans l'UE a diminué depuis la fin de 2001 et diminuera probablement encore durant les prochaines années. Cette situation est principalement due à une baisse de la production d'aliments composés, à la réduction des marges de sécurité en formulation, à des recommandations d'apport alimentaire en phosphore plus basses, et à l'utilisation croissante des phytases pour les aliments destinés aux monogastriques. L'interdiction des farines animales dans les aliments de bétail en 2000 avait entraîné une augmentation 
Figure 2. La production de phosphate bicalcique dihydraté avec l'acide chlorhydrique.

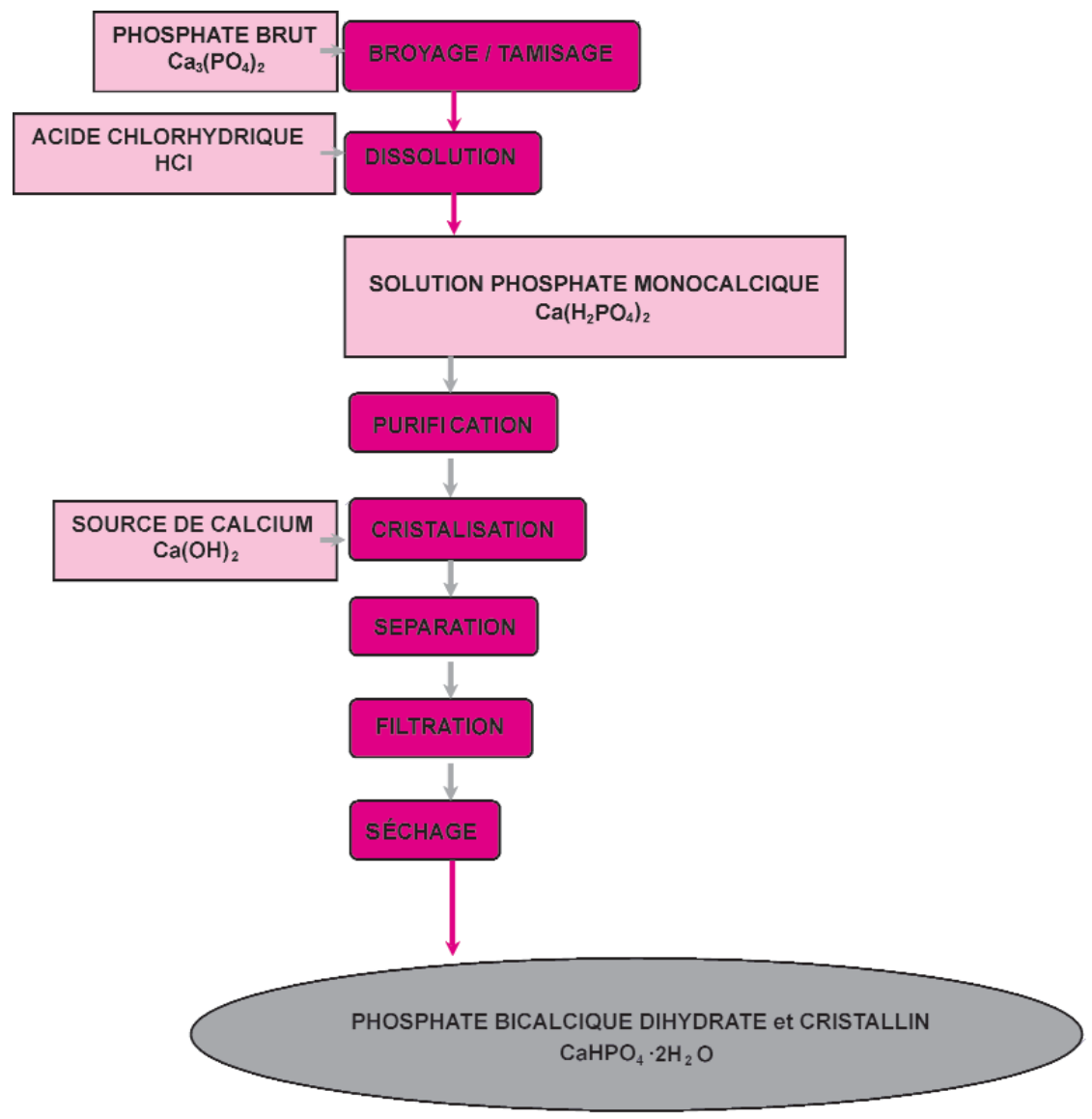

Figure 3. La production de phosphate monocalcique monohydrate avec l'acide phosphorique.

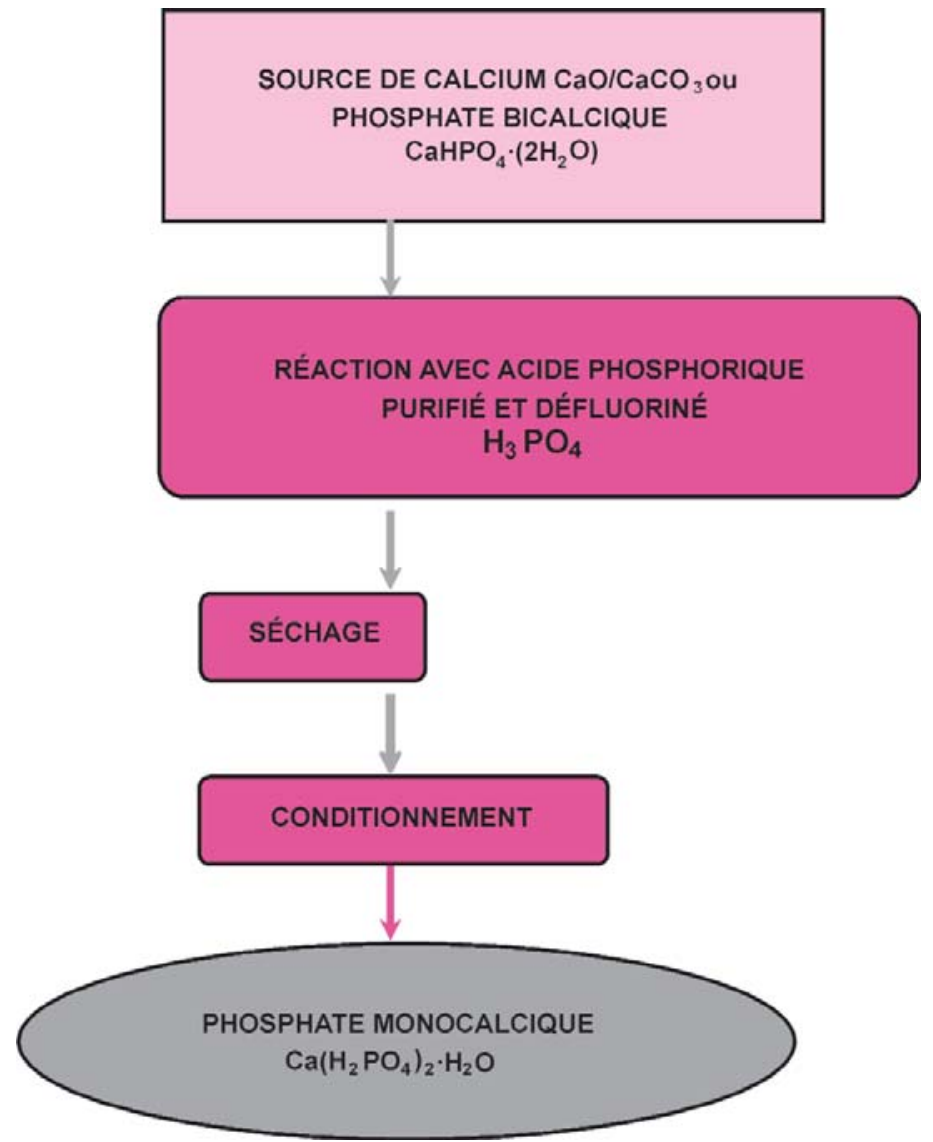

de la capacité de production de phosphates alimentaires sur le marché européen. Les effets combinés de ces deux évolutions font que, actuellement, les producteurs des phosphates alimentaires dans l'UE n'utilisent que 60 à $65 \%$ de leur pleine capacité de production.

\section{3 / Critères de qualité des phosphates alimentaires}

La productivité importante de l’élevage, les exigences des professions de l'industrie de l'alimentation animale, la pression environnementale et la législation européenne sévère, font que la demande de l'alimentation animale s'oriente vers des phosphates alimentaires de bonne qualité nutritionnelle. Les critères de sélection pour de tels produits sont présentés au tableau 1. Les phosphates alimentaires de haute qualité doivent être élaborés dans un environnement industriel et selon des procédés de fabrication strictement contrôlés. Le choix des matières premières et leur conformité à un cahier des charges précis sont à la base d'un résultat de qualité permettant la mise sur le marché de phosphates alimentaires stables présentant des variations de composition aussi faibles que possible. Les produits finis doivent subir un contrôle analytique régulier et rigoureux, et dans un souci de transparence, ces résultats devraient être toujours à la disposition des utilisateurs.

La solubilité dans l'eau et l'acide citrique à 2 \% d'un phosphate alimentaire est, surtout en France, utilisée comme indicateur de sa digestibilité. La solubilité du phosphore d'un phosphate de bonne qualité doit atteindre au moins à $95 \%$ dans l'eau et l'acide citrique à $2 \%$ et dans le citrate d’ammonium alcalin. La solubilité du phosphore dans l'eau des phosphates monobicalcique ou monocalcique (indicateur de pureté chimique de ces produits) devrait atteindre des niveaux suffisamment élevés, soit respectivement $50 \%$ et $80 \%$.

Lors de la fabrication d'aliments minéraux, le démélange doit être évité. Ceci est possible en sélectionnant des ingrédients minéraux sur leurs propriétés physiques telles que la densité et la granulométrie. Par conséquent, il est important que les phosphates alimentaires utilisés, soient conformes à leurs spécifications afin d'éviter le risque d’hétérogénéité des mélanges de minéraux ayant pour résultat des produits de 
Tableau 1. Les caractéristiques des phosphates alimentaires de haute qualité.

\begin{tabular}{|c|c|}
\hline Des produits stables et contrôlés & 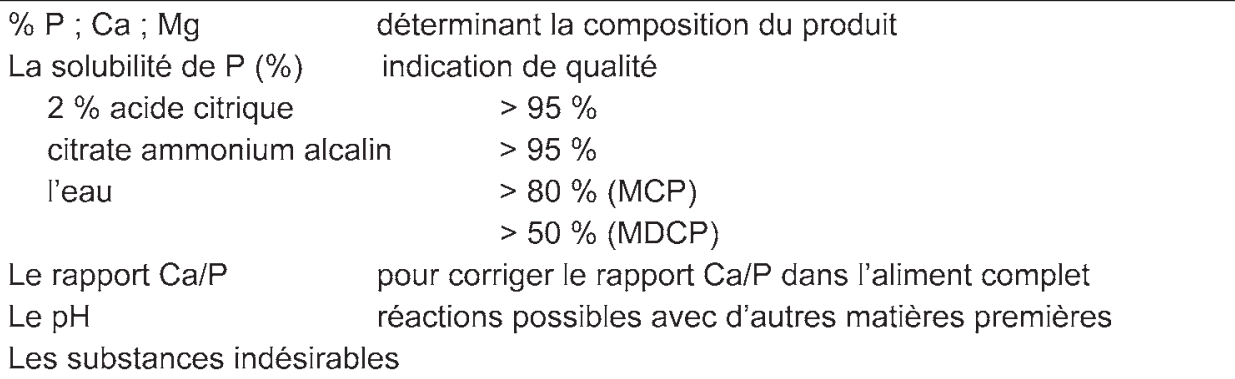 \\
\hline $\begin{array}{l}\text { Une digestibilité connue } \\
\text { Une digestibilité élevée }\end{array}$ & $\begin{array}{l}\text { Déterminé avec une technique «in vivo» } \\
\text { Dépendant de l'animal } \\
\text { Evaluation des phosphates basés sur la teneur en phosphore digestible plutôt qu'en } \\
\text { phosphore total } \\
\text { Réduction d'excrétion de phosphore dans l'environnement }\end{array}$ \\
\hline L'assurance de qualité & ISO 9001-2000 / HACCP / GMP \\
\hline La traçabilité des produits & La sécurité des aliments du bétail \\
\hline
\end{tabular}

qualité insuffisante pour répondre aux exigences alimentaires de l’animal.

Un autre paramètre important à prendre en compte est la réactivité des phosphates alimentaires. Celle-ci doit être la plus faible possible afin d'éviter la dégradation d'autres substances comme les vitamines, les acides aminés, les enzymes... durant la fabrication des aliments minéraux.

Les niveaux maxima tolérés de substances indésirables dans les phosphates alimentaires sont réglementés dans l'UE (tableau 2). Il est évident que tous les phosphates alimentaires de qualité doivent être conformes à cette législation. Pour répondre à ces exigences réglementaires, non seulement les produits finaux, mais également les matières premières, utilisées pour la production des phosphates alimentaires, doivent être contrôlées à des intervalles réguliers.

En résumé, les phosphates alimentaires de haute qualité sont caractérisés à la fois par une teneur élevée en phosphore, en calcium et/ou en magnésium, des solubilités in vitro élevées, par une faible teneur en substances indésirables et par des variations minimes de leurs propriétés physiques.

\section{4 / Les phosphates alimen- taires de digestibilité élevée}

La Commission Européenne a édité en juillet 2003 un document de référence sur les meilleures techniques disponibles, Best Availables Technics (EIPPCB 2003) pour l'élevage intensif des volailles et des porcs (http://eippcb.jrc.es). Une des recommandations proposée pour réduire l'excrétion du phosphore, tout en assurant la couverture des besoins des animaux monogastriques sans préjudice pour leur santé et leur production, consiste à utiliser des aliments complets contenant des phosphates alimentaires ayant une digestibilité élevée. La directive 98/67/EC de la Commission (chapitre 11, partie B) fait référence à plusieurs types de phosphates alimentaires utilisés dans l'UE. Ils diffèrent quant à leur teneur en minéraux, leur composition chimique et leur digestibilité. L'uti-

Tableau 2. La législation européenne concernant les éléments indésirables.

\begin{tabular}{|l|l|}
\hline \multicolumn{1}{|c|}{$\begin{array}{c}\text { Législation } \\
\text { Eléments indésirables }\end{array}$} & \multicolumn{1}{c|}{$2003 / 100 / E C$} \\
\hline Arsenic & Max $10 \mathrm{ppm}$ \\
Cadmium & Max $10 \mathrm{ppm}$ \\
Plomb & Max $15 \mathrm{ppm}$ \\
Mercure & Max $0,1 \mathrm{ppm}$ \\
Fluorine & Max 0,2\% \\
& $2003 / 57 / \mathrm{EC}$ \\
Dioxines (ng WHO-PCDD/F-TEQ/kg) & Max $1 \mathrm{ng} / \mathrm{kg}$ \\
\hline
\end{tabular}

lisation de phosphates alimentaires de bonne digestibilité aura un impact favorable sur l'environnement en limitant les quantités de $\mathrm{P}$ excrétées, un exemple est donné dans le tableau 3. Pour les volailles, il y a un avantage environnemental substantiel à employer des phosphates alimentaires fortement digestibles, comme le monocalcique. Des résultats comparables peuvent être obtenus pour les porcs. Le tableau 4 regroupe des données de digestibilité chez les volailles et les porcs, la hiérarchie est comparable chez les deux espèces, même si les valeurs absolues diffèrent parfois. Il est également à noter que deux phosphates bicalciques anhydres figurent dans ce tableau, ils présentent une différence de teneur en phosphore, due à leur origine industrielle. La digestibilité du phosphate monocalcique est la plus élevée, suivie de celles des phosphates monobicalcique et bicalcique. Cependant, il convient de souligner que le phosphate bicalcique dihydraté (DCP2 $\left.\mathrm{H}_{2} \mathrm{O}\right)$ a une digestibilité plus élevée que celle du phosphate bicalcique anhydre $\left(\mathrm{DCP} O \mathrm{H}_{2} \mathrm{O}\right)$. Le phosphate naturel défluoré (DFP) présente une digestibilité nettement inférieure.

Afin de répondre avec plus de précision aux besoins des animaux, mais également au point de vue environnemental, il est important de formuler les aliments du bétail sur la base du phosphore digestible et non du phosphore total. La figure 4 montre que pour trois phosphates différents, le DCP2 $\mathrm{H}_{2} \mathrm{O}$, le $\mathrm{DCPOH}_{2} \mathrm{O}$ et le DFP, ayant la même teneur en phosphore total (180 g/kg), l'apport en P digestible sera sensiblement différent ainsi 
Tableau 3. Réduction estimée d'excrétion de phosphore ${ }^{1}$.

\begin{tabular}{|l|c|c|c|c|c|}
\hline $\begin{array}{c}\text { Phosphates } \\
\text { alimentaires }\end{array}$ & $\begin{array}{c}\text { Digestibilité volailles } \\
\text { (\%) }\end{array}$ & $\begin{array}{c}\text { Taux d'incorporation } \\
(\%)\end{array}$ & $\begin{array}{c}\text { Taux d'incorporation } \\
\text { (g P) }\end{array}$ & $\begin{array}{c}\mathbf{g} \mathbf{P} \\
\text { absorbé }^{2}\end{array}$ & $\begin{array}{c}\mathbf{g} \mathbf{P} \\
\text { excrété }^{2}\end{array}$ \\
\hline DFP / CaNaP & 59 & 1,56 & 28 & 16,5 & 11,5 \\
MCP & 84 & 0,87 & 19,6 & 16,5 & 3,1 \\
\hline
\end{tabular}

1 Calcul basé sur la digestibilité des volailles.

2 Originaire d'un phosphate alimentaire inorganique.

3 Van der Klis et Versteegh (1996).

Figure 4. La teneur en phosphore digestible de 3 phosphates alimentaires avec la même teneur en phosphore total.

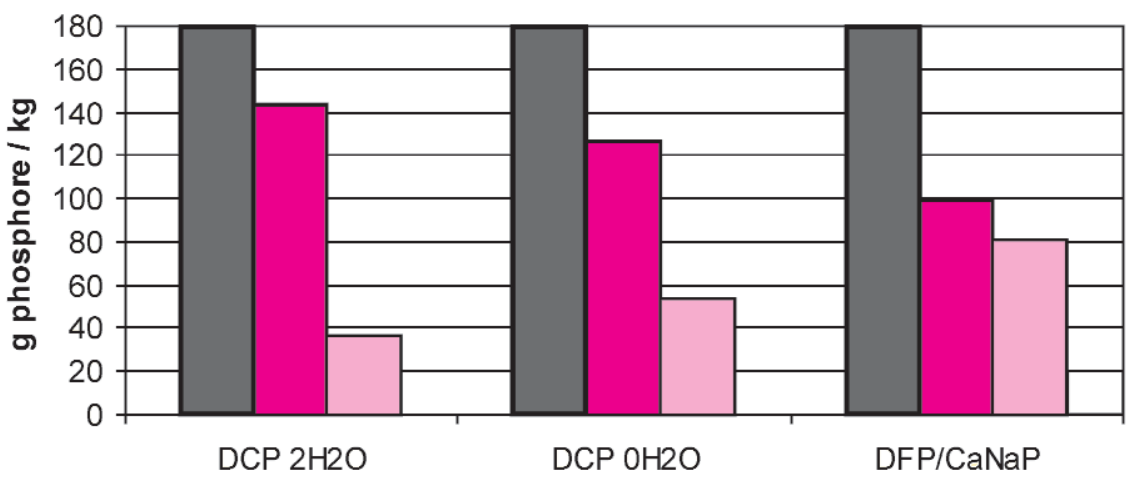

$\mathrm{P}$ total $\square \mathrm{P}$ digestible $\square \mathrm{P}$ excrété

les animaux recevant le DFP ne disposeront que de $68 \%$ de l'apport réalisé par le DCP2 $\mathrm{H}_{2} \mathrm{O}$, avec des conséquences prévisibles.

Il est donc important de formuler l'alimentation des animaux monogastriques sur base du système de phosphore digestible pour les porcs (Jondreville et al 2005)) ou disponible pour les volailles (Lescoat et al 2005) et d'employer des sources complémentaires ayant une digestibilité élevée pour optimiser les performances des animaux dans le respect des contraintes environnementales.

\section{5 / La garantie de qualité et traçabilité des produits}

Le 12 janvier 2005 a été publié le règlement $n^{\circ} 183 / 2005 \mathrm{du}$ parlement européen et du conseil établissant des exigences en matière d'hygiène des aliments pour animaux. Les deux éléments principaux de ce règlement sont la garantie de la qualité par un système de HACCP (Hazard Analysis and Critical Control Point System) et par un code de bonnes pratiques (GMP = Good Manufacturing Practice) et de traçabilité des produits. Cette dernière peut être garantie par un contrôle régulier de toutes les matières premières et par la surveillance et le contrôle de la production. Tous les produits finaux, en vrac, bigbag ou sacs, doivent être analysés et recevoir un numéro du lot. Lors de chaque expédition, des échantillons doivent être prélevés et gardés pendant plusieurs mois. De cette façon, les fabricants de phosphates alimentaires peuvent garantir la sécurité et la traçabilité complète de leur production.

\section{Conclusion}

Les phosphates alimentaires de haute qualité sont définis par des caractéristiques physico-chimiques précises et stables, par des solubilités in vitro du phosphore élevées et par des teneurs en substances indésirables aussi basses que possible. De plus, ces phosphates sont caractérisés par une digestibilité du phosphore élevée réduisant les rejets de $\mathrm{P}$ dans le milieu ambiant, sans compromettre les performances et l'état de santé des animaux. Les phosphates alimentaires de qualité sont produits par des entreprises ayant mis en place des procédures de traçabilité et de contrôles de qualité. De cette façon, ces phosphates contribuent à apporter la qualité «de l'étable à la table».

Tableau 4. La teneur en phosphore digestible (= phosphore total x \% digestibilité) des phosphates alimentaires différents.

\begin{tabular}{|c|c|c|c|c|c|}
\hline Phosphates alimentaires & & \multicolumn{2}{|c|}{ Volailles } & \multicolumn{2}{|c|}{ Porc } \\
\hline & $g P$ total $/ \mathrm{kg}$ & digestibilité \% & g P digestible & digestibilité \% & g P digestible \\
\hline$M C P$ & 229 & 84 & 195 & 90 & 206 \\
\hline$M D C P$ & 219 & 83 & 182 & 80 & 175 \\
\hline$D C P 2 \mathrm{H}_{2} \mathrm{O}$ & 182 & 80 & 146 & 72 & 131 \\
\hline$D C P \mathrm{OH}_{2} \mathrm{O}$ & 202 & 70 & 141 & 65 & 131 \\
\hline$D C P \mathrm{OH}_{2} \mathrm{O}$ & 180 & 70 & 126 & 65 & 117 \\
\hline DFP/CaNaP & 180 & 59 & 99 & 60 & 108 \\
\hline
\end{tabular}

Sources : Lippens et Huyghebaert, 2003, CVB, 2004, Kemme et al 2002, Eeckhout et De Paepe, 1997, Tessenderlo Group. 


\section{Références}

CVB, Centraal Veevoederbureau, Veevoe dertabel 2004. ISBN 90-72839-13-7.

Eeckhout W., De Paepe M., 1997. The digestibility of three calcium phosphates for pigs as measured by difference and by slope-ratio assay. J. Anim. Physiol. A. Anim. Nutr., 77, 53-60.

EIPPCB, European Integrated Pollution Prevention and Control Bureau, 2003. Reference Document on Best Available Techniques for Intensive Rearing of Poultry and Pigs. http://eippcb.jrc.es.
Jondreville C., Dourmad J.Y., 2005. Le phosphore dans l'alimentation des porcs. INRA Prod. Anim. 18, 183-192.

Kemme P.A., Kwakernaak C., van der Klis J.D., 2002. The absorbability of phosphorus in feed phosphates for broilers. Aliphos ${ }^{\circledR}$ Monocal, Italphos ${ }^{\circledR}$ Monocal and Italphos ${ }^{\circledR}$ Dical 18 . Confidential Report ID TNO Animal Nutrition $n^{\circ} 2188$.

Lescoat P., Travel A., Nys Y., 2005. Lois de réponse des volailles de chair à l'apport de phosphore. INRA Prod. Anim., 18, 193-201.

Lippens M., Huyghebaert G., 2003. Comparison of two different phosphate sources in a balance trial. Confidential report CLO Department of Animal Nutrition and Husbandry.

Van der Klis J.D., Versteegh H.A.J., 1996. Phosphorus nutrition of poultry. In: Recent advances in animal nutrition, Nottingham University Press, 309-320.

\section{Résumé}

La consommation des phosphates alimentaires dans l'Union Européenne a diminué depuis la fin de 2001 suite à la baisse de la production d'aliments composés ; à la prise en compte de marges de sécurité plus faibles en formulation et des normes d'apport alimentaire réduites en phosphore, et d'une utilisation croissante de la phytase. Face à cette situation, la réponse de l'industrie des phosphates alimentaires devrait être centrée sur la qualité nutritionnelle des produits.

Les phosphates alimentaires de haute qualité sont caractérisés par des propriétés physico-chimiques stables et précises, par une bonne solubilité du phosphore et par une teneur aussi basse que possible en substances indésirables. Ces phosphates sont en outre caractérisés par une digestibilité élevée du phosphore réduisant au minimum le phosphore excrété dans l'environnement sans mettre en danger la santé, la production et le bien-être des animaux. En outre, seules les sociétés disposant d'une certification de garantie de qualité et de systèmes de traçabilité mis en application peuvent assurer une production de phosphates alimentaires de haute qualité, assurant la sécurité de l'alimentation animale. De cette façon, les phosphates alimentaires contribuent à apporter la qualité «de l'étable à la table» dans l’Union Européenne.

\section{Abstract}

\section{Characteristics of nutritional of feed phosphates}

The consumption of feed phosphates in the European Union has been decreasing since the end of 2001 due to a decreasing production of compound feed; changing feed formulations with lower safety margins and reduced phosphorus norms, and by a large increase in the use of phytase. The response of the phosphate industry to decreasing phosphate consumption in the European feed industry should be quality.

High quality feed phosphates are characterised by stable and accurate physical-chemical properties, good phosphorus solubility and a low amount of undesirable substances. Such phosphates are certainly characterised by a high phosphorus digestibility minimising the phosphorus output into the environment without endangering the health, the performance and welfare of the animals. In addition, only companies with Quality Assurance certification and implemented traceability systems can guarantee to produce consistently high quality feed phosphates, ensuring feed safety. In this way, feed phosphates contribute to bringing quality "from the stable to the table" in the European Union.

BLEUKX W., 2005. Production et qualité nutritionnelle des phosphates alimentaires. INRA Prod. Anim., 18, $169-173$. 
